Review

\title{
Selective Chromatid Segregation Mechanism Invoked For the Human Congenital Mirror Hand Movement Disorder Development by RAD5 I Mutations: A Hypothesis
}

\begin{abstract}
Amar J. S. Klar $\bowtie$
Gene Regulation and Chromosome Biology Laboratory, National Cancer Institute, Center for Cancer Research, National Institutes of Health, Building 539, Room 154, Frederick, MD 21702-1201, USA.

$\triangle$ Corresponding author: A. K., Gene Regulation and Chromosome Biology Laboratory, National Cancer Institute, Center for Cancer Research, National Institutes of Health, Frederick, MD 21702-1201, USA. Email: klara@mail.nih.gov Phone 3018465916 Fax 3018466911.

(c) Ivyspring International Publisher. This is an open-access article distributed under the terms of the Creative Commons License (http://creativecommons.org/ licenses/by-nc-nd/3.0/). Reproduction is permitted for personal, noncommercial use, provided that the article is in whole, unmodified, and properly cited.
\end{abstract}

Received: 2014.06.12; Accepted: 2014.07.12; Published: 2014.09.10

\begin{abstract}
The vertebrate body plan externally is largely symmetrical across the midline but internal organs develop asymmetrically. The biological basis of asymmetric organ development has been investigated extensively for years, although the proposed mechanisms remain controversial. By comparison, the biological origin of external organs symmetry has not been extensively investigated. Bimanual hand control is one such external organs symmetry allowing independent motor control movements of both hands to a person. This gap in our knowledge is illustrated by the recent reports of heterozygous rad5 I mutations causing mysterious symptoms of congenital mirror hand movement disorder (MM) in humans with $50 \%$ penetrance by an unknown mechanism. The analysis of mutations that vary symmetry or asymmetry could be exploited to decipher the mechanisms of laterality development. Here I present a hypothesis for explaining $50 \%$ penetrance of the rad5 I mutation. The MM's origin is explained with the Somatic Strand-specific Imprinting and selective sister chromatid Segregation (SSIS) hypothesis proposed originally as the mechanism of asymmetric cell division to promote visceral organs body plan laterality development in vertebrates. By hypothesis, random sister chromatid segregation in mitosis occurs for a specific chromosome due to rad5I/RAD5 I constitution causing MM disorder development in $50 \%$ of subjects.
\end{abstract}

Key words: Mirror hand movement disorder, Selective chromatid segregation mechanism, Brain laterality development, rad51 mutation etiology, Asymmetric cell division mechanism.

\section{RAD51 haploinsufficiency causes invol- untary mirror hand movements disorder in humans}

Human hand movements are bimanual such that one hand can be intentionally used independently from the other. Here I designate this behavior to be an example of body symmetry for external body structures in which either hand can be used independently. Interestingly, there is a congenital human mirror movement disorder (MM [MIM 157600]) that causes involuntary movements of one hand, which mirrors the intentional movements of the other hand. The disorder is an extremely rare due to genetically inherited trait, although sporadic cases of unknown etiology also exist. Curiously, the condition in familial cases is associated with heterozygous rad51 mutation discovered in three independent families. Notably, 8 of the 16 heterozygous rad51 nonsense mutation-carriers in one family developed the disorder (2). The transmission pattern is consistent with autosomal dominant inheritance with incomplete $(50 \%)$ pene- 
trance in one family.

The hereditary MM disorder is also caused by autosomal dominant mutations in the DCC (deleted in colorectal carcinoma) gene $(5,6)$. DCC/netrin- 1 signaling gene mutations because abnormal ipsilateral axonemal nerve fiber connections, therefore, how $d c c$ mutations cause the disorder is clearly understood. In vivo and in vitro experiments support the hypothesis that gradients of netrin- 1 protein in the developing nervous system cause axons extension by using a DCC receptor protein (7). Importantly, it was recently reported that the same neuronal circuits that are affected by $d c c$ mutations are similarly affected in rad51/RAD51 subjects having the disorder (3). Specifically, abnormal uncrossed corticospinal tract and abnormal bilateral primary motor cortices activation during manual tasks is found in MM patients. Clearly, these two different genes are required for the development of motor control. The MM behavior in humans predominates in the upper limbs affecting the muscles of the hands and fingers, although lower limbs might be affected as well in some subjects. In comparison, $d c c$ deletion mutations in mice, aptly named Kanga mice, inflict their lower limbs resulting in a distinctive hopping gait instead of the normal walk (8).

In contrast to DCC mutations, how rad51 mutations cause defective motor control development is not understood. The authors proposed two possibilities regarding the RAD51 function: one postulates a role in apoptosis during early development of the central nervous system and the other postulates that RAD51 might have a role in axonal guidance of developing neurons. The RAD51-deficiency caused disorder raises interesting questions concerning the basis of the disorder and knowing it has implications for how bimanual laterality is normally specified. The RAD51 protein is well known for its function in repairing double-stranded DNA breaks in chromosomes (9). Therefore, how could defect in a recombination factor cause the disorder? What is the basis of the $50 \%$ penetrance of the RAD51 mutation? The absence of the MM disorder in $50 \%$ of genetically predisposed carriers was speculated to be due to higher expression of RAD51 from the normal allele, or due to other genetic or epigenetic modifiers (2). Here a fundamentally different kind of hypothesis is advanced to explain the $50 \%$ disease penetrance result.

\section{The SSIS model proposed to account for asymmetric or symmetric cell division during development}

One central question of biology addresses mechanisms that produce developmentally equivalent or nonequivalent daughter cells at specific cell divisions during development and tissue homeostatis. A unique mechanism of asymmetric cell division in the fission yeast Schizosaccharomyces pombe has been described: it is based on the synthesis of epigenetically non-equivalent sister chromatids at the mating-type locus during chromosome replication $(10,11)$. In yeast, the inherent chirality of strands of the double-helical structure of DNA (12), unidirectional replication of the mating-type locus along with the DNA strands replication history provide the primary bases for sister cell differentiation in this single-cell, haploid organism by epigenetic differentiation of sister chromatids (reviewed in (13)). Similarly, developmentally important genes might be differentially regulated by somatically installing heterochromatin assembly in a strand-specific fashion to produce differentiated sister chromatids at specific stages in development of diploid organisms. It is known that active versus silenced epigenetic states of gene expression are very stable and can be inherited as classical Mendelian/chromosomal markers in mitosis and in meiosis in fission yeast $(14,15)$. To exploit such an epigenetic mechanism, both asymmetric and symmetric cell division mechanisms depending on the specific mode of sister chromatids distribution may have evolved to drive development (Figure 1). For example, by inducing site-specific chromosome 7 recombination, the denominated selective $\mathrm{W}, \mathrm{W}:: \mathrm{C}, \mathrm{C}$ segregation of strands/chromatids was found in endoderm and embryonic stem cell mitoses, another selective W, C : $\mathrm{W}, \mathrm{C}$ segregation pattern was noted in neuroectoderm cell divisions, but a random chromatid distribution occurred in pancreatic, mesoderm and cardiomyocyte mouse cells (16). To help define the mechanism of selective segregation process, both nonrandom modes changed to random mode when the left-right dynein $(L R D)$ gene was inactivated by the RNAi technology $(17,18)$. Note that the LRD gene is famous for controlling visceral organs laterality distribution to the left- versus right-body side in mice $(19,20)$. Also, a Drosophila study recently reported an 85:15 ratio of biased sex chromosome's chromatid segregation during male germline stem cell division by employing the technique of chromosome orientation fluorescence in situ hybridization (21). The significance of such biased segregation phenomenon remains unknown. Amazingly, autosomal chromatids in this Drosophila study segregated only with the selective $\mathrm{W}, \mathrm{W}:: \mathrm{C}, \mathrm{C}$ mode (22). These studies show that cell-type regulated, chromosome-specific, biased, selective or random sister chromatid segregation mechanisms operate in evolutionarily diverse organisms, as postulated in the SSIS model (Figure 1). A possible biological function of the selective chromatid segregation mechanism for eukaryotic development is explored in this paper. 


\section{The SSIS mechanism invoked to explain the MM syndrome of rad51 heterozygote individuals}

In this communication, the biological basis of symmetrical contralateral motor hand control development is addressed. I propose that the brain hemispheres producing progenitor cell divides symmetrically during embryogenesis (Figure 2). Specifically, it is proposed that the RAD51 protein functions to cause $\mathrm{W}, \mathrm{C}: \mathrm{W}, \mathrm{C}$ segregation of a specific chromosome to result in symmetric cell division. In this manner, axonal crossing occurs at the midline causing the de-

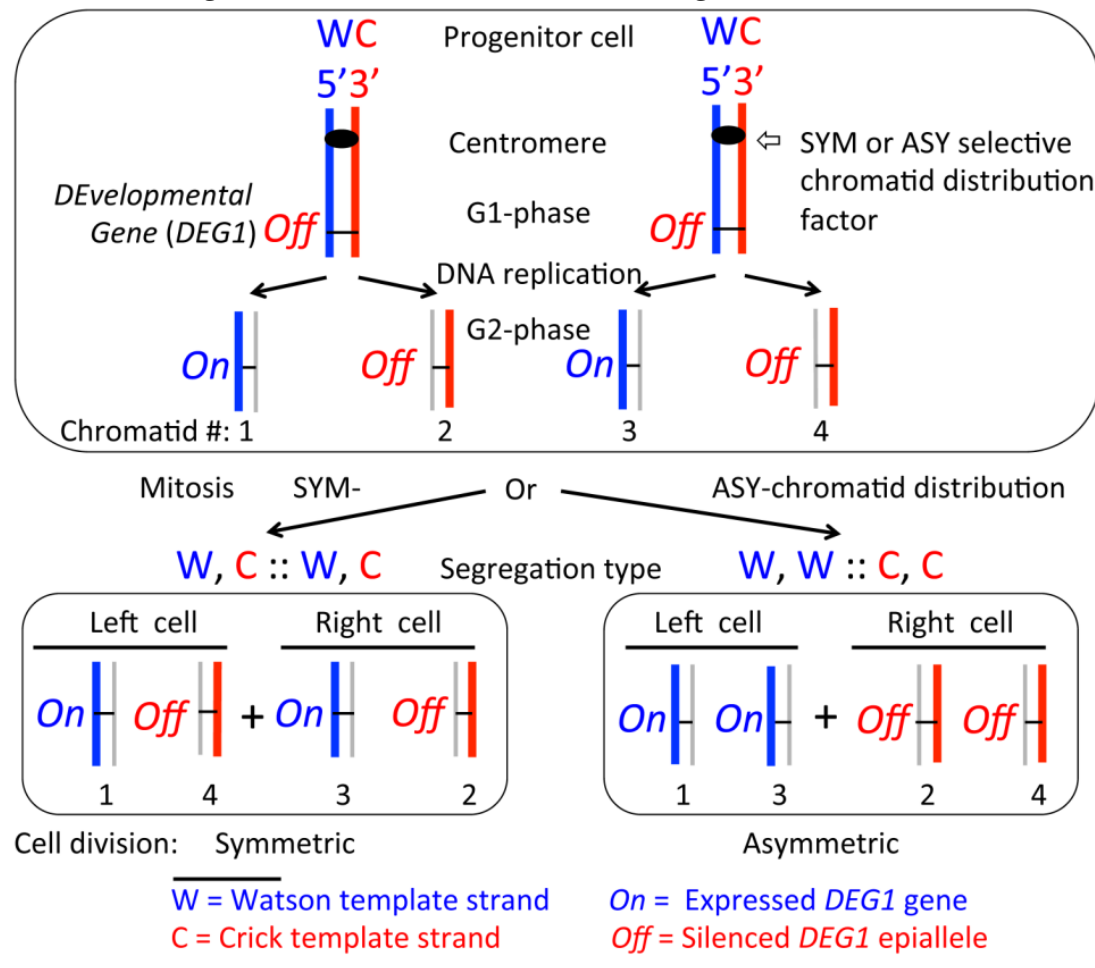

Figure I: The SSIS model proposed to account for asymmetric or symmetric cell divisions during development. The model $(4,23)$ makes the following three postulates: $(A)$ the progenitor cell carries the hypothetical DEvelopmental Gene I (DEGI) epigenetically silenced (Off) on both homologs of a chromosome; (B) DEGl is activated (On) during replication in the template W (arbitrarily colored blue) strand-containing chromatid at a specific cell division, somewhat like the HOXB gene expression requires DNA replication of cells of specific cell type during mouse embryogenesis (24), but the template $C$ (red colored) strand-containing chromatid maintains the off state just like the parental cell; and (C) the progenitor cell will divide in such a way to selectively segregate specific chromatids to the resulting left- versus right-side placed daughter cells with respect to predetermined embryonic anterior-posterior and dorso-ventral axes of the embryo. In principle, two types of selective segregation modes could evolve: (I) a hypothetical symmetry-specifying factor (SYM) dictates the denominated W, C :: W, C (by referring to template strands) chromosome segregation mode by functioning at centromeres of the specific chromosome to deliberately produce symmetric cell division; (II) a hypothetical asymmetry-specifying factor (ASY) causes the W, W :: C, C segregation to produce an asymmetric cell division. Then, differential regulation of a key gene(s) in daughter cells could start a cascade of downstream-regulated events to promote symmetric or asymmetric mode of body laterality development. For example, thus differentiated daughter cells' progeny could generate lateralized body organs during development. The $W$ and $C$ strands are defined through their DNA sequence, 5 ' to 3' chemical polarity and replication history. To appreciate segregation mode, all template strands are represented by thicker lines while thinner lines in grey color represent those synthesized in the present replication cycle in the progenitor cell. The numbers I to 4 indicate specific chromatids inheriting indicated DNA strands by replication of a pair of homologous chromosomes. Multiple chromosomes could be also subjected to the SSIS process when multiple developmental genes are simultaneously regulated epigenetically to accomplish cellular differentiation. The SSIS model provides molecular basis for cellular differentiation owing to the double helical structure of DNA. velopment of contralateral and independent hand-movement control. In the MM disorder, random sister chromatid segregation is proposed to occur due to the reduced level of RAD51 or due to an abnormal rad51/RAD51 protein complex present in heterozygous individuals (Figure 2). As a result of the random chromatid distribution, 50\% rad51/RAD51 individuals would be unaffected because of the usual W, C :: W, C segregation had occurred in them by chance and an asymmetric cell division would occur in the remaining $50 \%$ persons leading to the disorder because of an abnormal homolateral motor control development. I MM phenotype and the $50 \%$ disorder penetrance of the rad51 mutation. The SSIS model is the only one postulating brain hemisphere to result from a single decision taken at a single, specific cell division and concerns a single chromosome segregation during embryogenesis in humans, and therefore, alteration of this control would not lead to other developmental anomalies. The observed $50 \%$ penetrance of rad51 heterozygote individuals is consistent with the single cell division decision postulated in our model. Moreover, rare sporadic MM cases may result from rare spontaneous mitotic recombination events occurring in the relevant chromosome of the progenitor cell (Figure 2).

\section{Experimental support for postulates of the SSIS model}

Because of technical difficulties, it has not been possible to test whether SSIS mechanism operates in development of higher eukaryotes. Thus far, only the cell mating systems of evolutionarily diverse fission yeasts S. pombe (10) and Schizosaccharomyces. japonicus (25) have provided examples in which epigenetically differentiated sister chromatids constitute the mechanism of asymmetric cell division. Concerning the related issue of visceral organs laterality development, directional liquid flow driven by primary cilia in the embryonic node structure or asymmetric expression of ion channels in sister cells are other models ad- 


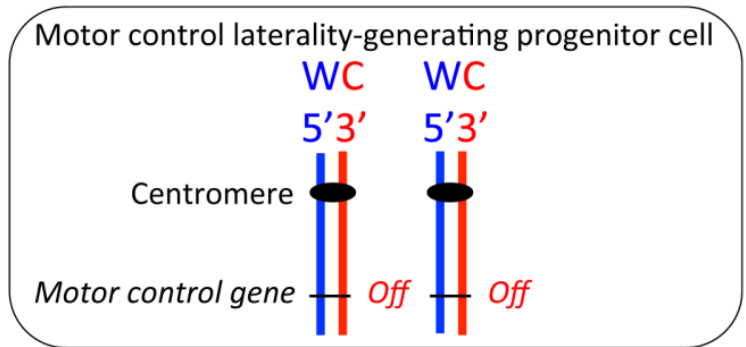

Random chromatid segregation in mitoses of rad51/RAD51 embryo

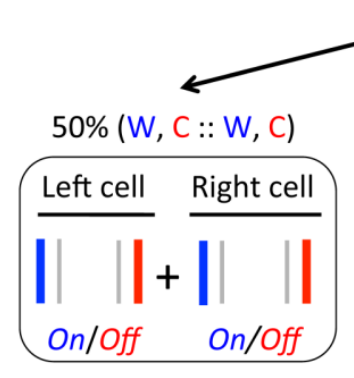

Symmetric cell division

$\vdash$ Normal subjects $\longrightarrow$

Figure 2: The SSIS model postulated to explain the MM syndrome's phenotypes. When the hypothetical motor control gene in chromatids is epigenetically regulated as diagrammed, followed by the W, C :: W, C chromatids segregation, humans develop the standard contralateral bimanual hand control (not shown). However, when the postulated random chromatid segregation occurs due to rad5I/RAD5I heterozygosity, only $50 \%$ of the subjects would develop the disorder. All other symbols used here are defined in the legend of figure 1.

vanced, however, the basis of breaking early left-right body symmetry during embryogenesis in vertebrates has remained controversial (1). The SSIS model was initially proposed as a mechanism for visceral organs laterality development in vertebrates $(4,17,19,20)$ and subsequently for the human brain laterality and psychoses development (23). One can imagine that the mechanisms that cause the development of left-right body asymmetry might be related to those causing bilateral symmetry, with both choices, in a sense, resulting from the alternative versions of the same developmental mechanism. The SSIS mechanism proposes bivalent precursor cell lineage (Figure 1) with the advantage of providing robustness to body laterality establishment because the parental cell produces daughter cells with precisely defined cell fates. In comparison, alternative development models propose superimposed symmetrical/asymmetrical lateralization by unknown mechanisms. Overall, the SSIS mechanism challenges the belief of that cell's fate is dictated somehow by the differential positioning of individual cells or groups of cells in the embryo.

The SSIS model proposes that offspring of a single progenitor cell generates the entire brain hemisphere, one cell giving rise to the left hemisphere and the other one giving rise to the right hemisphere. These progenitor cells are produced as sister cells at a specific cell division (Figures 1 \& 2). By the SSIS hypothesis, an asymmetric cell division early in development might establish a transcriptional cascade by activating a specific gene(s) in a cell, resulting in brain laterality development from its offspring. For example, the left-body-sided MI motor neuron and the right-sided eD3 epithelial cell in the Caenorhabditis elegans pharynx derive from the asymmetric division of a single blastomere cell dividing several cell divisions earlier in embryogenesis (26). This C. elegans study shows how cells can become committed during earlier developmental stages to specify an invariant cell fate later in development. Experimentally testing this single cell's progeny proposal has not been possible in mammals although brain hemispheric asymmetry is set, consistent with the SSIS model, at the 2-blastomere stage of the frog embryo (19).

In mammals, one of the $X$ chromosomes is inactivated, usually randomly in most cell divisions, due to the $X$ chromosome inactivation phenomenon. In contrast, a recent study (27) discovered that individual $X$ chromosomes of ectodermal cells remarkably maintain their transcriptionally active/inactive state during cell division, often during all stages of the developmental process. We propose that this feature can be exploited to fate map cells producing ectoderm-derived tissues, such as those of the entire brain hemisphere. By tagging one $X$ chromosome with a green florescent protein transgene marker and the other one with the red protein marker, this mouse study discovered that excitatory neurons of the entire brain hemisphere express primarily one of the two color markers. We surmise here that this result is consistent with the cell lineage proposal of the SSIS model, that is, the offspring of a single cell produce the entire brain hemisphere. These results are inconsistent with the most prominent morphogen-gradient model under investigation for many decades to explain eukaryotic development $(1,19)$.

\section{How might RAD51 function in SSIS mechanism and its deficiency cause MM disorder?}

The RAD51, a homolog of RecA of prokaryotes, is well known to function in DNA repair and recombination in chromosome arms. Curiously, RAD51 
binds to centromeres in S-phase, helps recombination but prohibits crossovers in the centromeres of $S$. pombe (9). Moreover, RAD51 is also required for cohesion of sister chromatids in mice (28). It is therefore possible that RAD51 promotes selective chromatid segregation in humans by maintaining centromere integrity and/or cohesion of sister chromatids. The SSIS hypothesis is designed here to explain the $50 \%$ penetrance of rad51 heterozygous mutations. Limited evidence supporting the three postulates of the SSIS model (Figure 1) have been only derived from diverse systems as discussed above, it is not known whether any of these phenomena operate for human brain development. However, because the prevailing theories (2) for explaining the role of RAD51 mutation in causing the MM disorder are not yet experimentally scrutinized, the SSIS hypothesis is proposed here as another one to consider for future research. Such a discussion is also aimed to highlight fascinating properties of the MM disorder as well as of the selective chromatid segregation mechanism. The thesis advanced here has implications for the elucidation of the molecular etiology of not yet understood human motor control development, and also to understand body laterality development in general, by building on amazing recent progress made in seemingly unrelated areas of research. One area that identified mutations causing the $\mathrm{MM}$ disorder by affecting brain hemispheric laterality development $(2,3)$ and another one that demonstrated the selective chromatid segregation mechanism operating in diverse organisms (16, $17,21,22)$. Following up on this discussion, it will be interesting to determine whether the language processing hemisphere of MM subjects varies from that found in unaffected subjects.

\section{Abbreviations}

MM, Congenital mirror hand movements syndrome; SSIS, Somatic Strand-specific Imprinting and selective sister chromatid Segregation model.

\section{Acknowledgments}

I thank my colleagues Donald Court and Sharon Moore and Mark Johnston (University of Colorado, Denver) for editorial suggestions on the manuscript. The Intramural Research Program of the National Cancer Institute, National Institutes of Health, supports this research.

\section{Competing Interests} exists.

The author declares that no competing interest

\section{References}

1. Vandenberg LN, Levin M. A unified model for left-right asymmetry? Comparison and synthesis of molecular models of embryonic laterality. Developmental biology. 2013; 379: 1-15.

2. Depienne C, Bouteiller D, Meneret A, et al. RAD51 haploinsufficiency causes congenital mirror movements in humans. Am J Hum Genet. 2012; 90: 301-307.

3. Gallea C, Popa T, Hubsch C, et al. RAD51 deficiency disrupts the corticospinal lateralization of motor control. Brain: a journal of neurology. 2013; 136: 3333-3346.

4. Klar AJS. A model for specification of the left-right axis in vertebrates. Trends Genet. 1994; 10: 392-396.

5. Srour M, Riviere JB, Pham JM, et al. Mutations in DCC cause congenital mirror movements. Science. 2010; 328: 592-592.

6. Depienne C, Cincotta M, Billot S, et al. A novel DCC mutation and genetic heterogeneity in congenital mirror movements. Neurology. 2011; 76: 260- 264.

7. de la Torre JR, Hopker VH, Ming GL, et al. Turning of retinal growth cones in a netrin-1 gradient mediated by the netrin receptor DCC. Neuron. 1997; 19: 1211-1224.

8. Finger JH, Bronson RT, Harris B, et al. The netrin 1 receptors Unc5h3 and Dcc are necessary at multiple choice points for the guidance of corticospinal tract axons. J Neurosci. 2002; 22: 10346-10356.

9. Nakamura KI, Okamoto A, Katou, et al. Rad51 suppresses gross chromosomal rearrangement at centromere in Schizosaccharomyces pombe. Embo J. 2008; 27: 3036-3046.

10. Klar AJS. Differentiated parental DNA strands confer developmental asymmetry on daughter cells in fission yeast. Nature. 1987; 326: 466-470.

11. Arcangioli B. A site- and strand-specific DNA break confers asymmetric switching potential in fission yeast. EMBO J. 1998; 17: 4503-4510.

12. Watson JD, Crick FH. Molecular structure of nucleic acids; a structure for deoxyribose nucleic acid. Nature. 1953; 171: 737-738.

13. Klar AJS, Ishikawa K, Moore S. A unique DNA recombination mechanism of the mating/cell-type switching of fission yeasts: a review. In: Craig NL, Chandler M, Gellert M, Lambowitz AM, Rice PA, Sandmeyers S, eds. Mobile DNA III. Washington, DC: American Society of Microbiology Press. 2014: in press.

14. Grewal SIS, Klar AJS. Chromosomal inheritance of epigenetic states in fission yeast during mitosis and meiosis. Cell. 1996; 86: 95-101.

15. Klar AJS. Propagating epigenetic states through meiosis: where Mendel's gene is more than a DNA moiety. Trends Genet. 1998; 14: 299-301.

16. Armakolas A, Klar AJS. Cell type regulates selective segregation of mouse chromosome 7 DNA strands in mitosis. Science. 2006; 311: 1146-1149.

17. Armakolas A, Klar AJS. Left-right dynein motor implicated in selective chromatid segregation in mouse cells. Science. 2007; 315: 100-101.

18. Armakolas A, Koutsilieris M, Klar AJS. Discovery of the mitotic selective chromatid segregation phenomenon and its implications for vertebrate development. Curr Opin Cell Biol. 2010; 22: 81-87.

19. Klar AJS. Support for the selective chromatid segregation hypothesis advanced for the mechanism of left-right body axis development in mice. Breast Disease. 2008; 29: 47-56.

20. Sauer S, Klar AJS. Left-right symmetry breaking in mice by left-right dynein may occur via a biased chromatid segregation mechanism, without directly involving the Nodal gene. Front Oncol. 2012; 2: 1-10.

21. Yadlapalli S, Yamashita YM. Chromosome-specific nonrandom sister chromatid segregation during stem-cell division. Nature. 2013; 498: 251-256.

22. Sauer S, Klar AJS. Reply to "Chromosome-specific nonrandom sister chromatid segregation during stem-cell division." Nature. 2013; 498: 254-256. It can be accessed online as a comment \#58907 of the Nature paper. http://www.nature.com/nature/journal/v498/n7453/full/nature12106.htm l\#comments.

23. Klar AJS. A genetic mechanism implicates chromosome 11 in schizophrenia and bipolar diseases. Genetics. 2004; 167: 1833-1840.

24. Fisher D, Mechali M. Vertebrate HoxB gene expression requires DNA replication. Embo J. 2003; 22: 3737-3748.

25. Yu C, Bonaduce MJ, Klar AJS. Defining the epigenetic mechanism of asymmetric cell division of Schizosaccharomyces japonicus yeast. Genetics. 2013; 193: 85-94.

26. Nakano S, Stillman B, Horvitz HR. Replication-coupled chromatin assembly generates a neuronal bilateral asymmetry in C. elegans. Cell. 2011; 147: 1525-1536.

27. $\mathrm{Wu} \mathrm{H}$, Luo $\mathrm{j}, \mathrm{Yu} \mathrm{H}$, et al. Cellular resolution maps of $\mathrm{X}$ chromosome inactivation: implications for neural development, function, and disease. Neuron. 2014; 81: 103-119.

28. Smeenk G, de Groot AJ, Romeijn RJ, et al. Rad51C is essential for embryonic development and haploinsufficiency causes increased DNA damage sensitivity and genomic instability. Mutation Research. 2010; 689: 50-58.

\section{Author biography}

Amar J. S. Klar obtained his Ph. D. under Professor Harlyn Halvorson from the University of Wisconsin at Madison in 1975. He did his postdoc training 
with Professor Seymour Fogel at the University of California at Berkeley from 1975 to 1978 . His postdoc work established the gene transposition model of mating-type switching of budding yeast and discovered the phenomenon of gene silencing in yeast by discovering the MAR1 gene (later named SIR2, Sirtuin) function in silencing the mating-type loci by epigenetics. He then held a faculty position at the Cold Spring Harbor Laboratory, becoming Director of the Delbruck Laboratory. There he worked on mating-type switching mechanism of both budding and fission yeasts from 1978 to 1988. Since 1988, the author has been heading the Developmental Genetics Section at NCI-Frederick facility in Maryland, USA. From the fission yeast work he discovered that asymmetric cell division results from differentiation of sister chromatids during DNA replication at the mating-type locus. It is this idea of sister chromatid differentiation and selective chromatid segregation he has advanced to promote visceral organs laterality development in mice, brain laterality, human hand-use preference, psychoses etiology in humans and for eukaryotic development in general. The present paper explains the biological origin of mirror hand movement syndrome in humans by his DNA strands chirality mechanism for developing lateralized brain hemispheres. Jeffrey Eugenides in 2012 immortalized his yeast cells sex change research in the best-selling novel, The Marriage Plot. 